\title{
Temperature Dependence of the Activation Volume of Secondary Relaxation in Glass Formers
}

\author{
Soheil Sharifi \\ Department of Physics, University of Sistan and Baluchestan, P.O. Box 98155-987 Zahedan, Iran \\ Correspondence should be addressed to Soheil Sharifi, soheil.sharifi@gmail.com
}

Received 5 April 2011; Accepted 27 May 2011

Academic Editors: R. Z. Domingues and N. G. Semaltianos

Copyright () 2011 Soheil Sharifi. This is an open access article distributed under the Creative Commons Attribution License, which permits unrestricted use, distribution, and reproduction in any medium, provided the original work is properly cited.

We study a nonmonotonic behavior with temperature of the activation volume $\Delta \mathrm{V}_{\beta}$ of secondary relaxation in PPGE [Poly[(phenyl glycidyl ether)-co-formaldehyde]] and PVAc [Poly(vinyl acetate)] glass formers. Our results show that the non-monotonic behaviour of secondary relaxation does not depend on the type of the secondary relaxation. Moreover, the study of secondary relaxation under pressure at isothermal paths shows two different behaviours of $\Delta \mathrm{V}_{\beta}$ in the glassy state (which describe two different types of glasses); for temperatures higher than the glass transition temperature $(\mathrm{Tg}), \Delta \mathrm{V}_{\beta}$ increases with a decrease of the temperature while at temperatures lower than $\mathrm{Tg}, \Delta \mathrm{V}_{\beta}$ decreases with a decrease of the temperature.

\section{Introduction}

The glassy state is a nonequilibrium state of materials [1]. As a consequence, the observed thermodynamic and relaxation properties slowly evolve with time because of the evolution of the molecular configuration towards a state with a lower energy [2]. Another consequence of the none-quilibrium condition of glasses is that the thermodynamic and relaxation properties show different values when measured in the glassy state at a fixed thermodynamic condition as a consequence of different vitrification histories [3-5]. For example, this was observed for the relaxation frequency of several secondary processes $[3,6]$ as a consequence of cooling with different rates, or after vitrification combining different sequences of cooling and compression steps [7-9]. Glass formers are characterized by a complex relaxation pattern, which evolves over several decades. Dielectric spectroscopy has proven particularly useful for studying such a scenario, as it is able to monitor the dielectric dynamics of a system over a range up to sixteen decades. It has been turned out that in such a broad dynamic range, several molecular processes take place, and most of them are usually characterized by nonexponential relaxation functions. In case that such processes depend on the local motion of hole molecule (intermolecular process), they are usually called the Johari-Goldstein relaxation (JG relaxation) which is a type of secondary relaxation $[10,11]$; otherwise they are called non-JG relaxation or intramolecular secondary relaxation. It has been suggested that the connection or the similarity of dynamic properties (dependence of relaxation time on temperature, pressure, thermodynamic history of glass formation) of the secondary relaxation with those of the structural one, can be used as a criterium to distinguish JG and non-JG relaxations [12]. However, the existence of such a connection is still questionable, and there is a debate about the validity of this criterium. A study of the pressure dependence of the JG relaxation of PPGE, along several isotherms, shows that $\Delta \mathrm{V}_{\beta}$ in the glassy state first increases and then slightly decreases on lowering $T$, reaching a maximum at the $T_{g}[13]$. This kind of temperature dependence occurs in the region of the $T_{g}$ at ambient pressure. A similar temperature dependence of the mean whole volume dispersion is proposed on the literature. Such an explanation supports previous work, suggesting that this process is of the JG type [14]. In this work, we present experimental studies varying pressure and temperature of secondary processes in two glass formers. Firstly, we study the temperature dependence of the activation volume of one observed secondary relaxation in PVAc and compare 


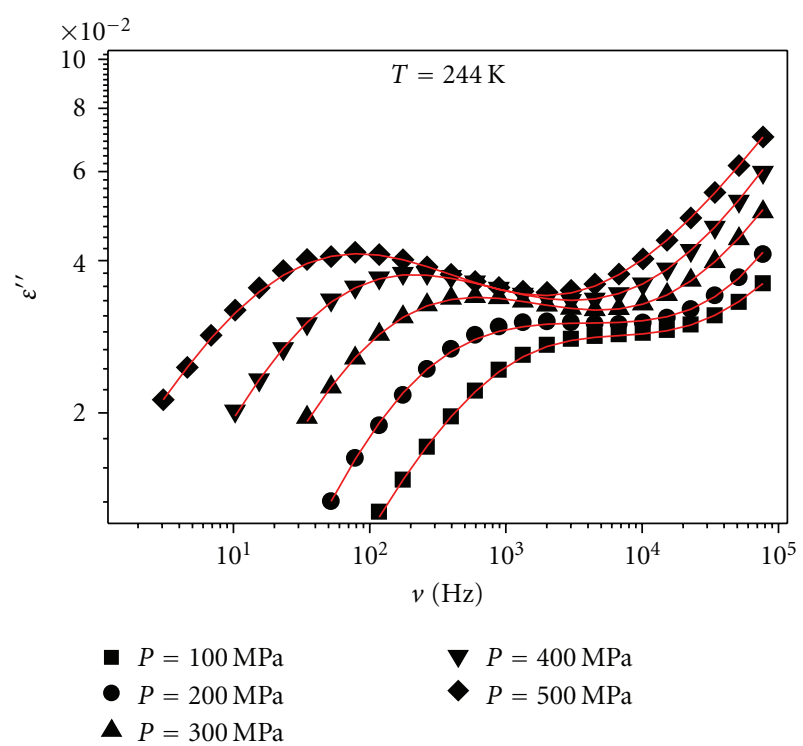

(a)

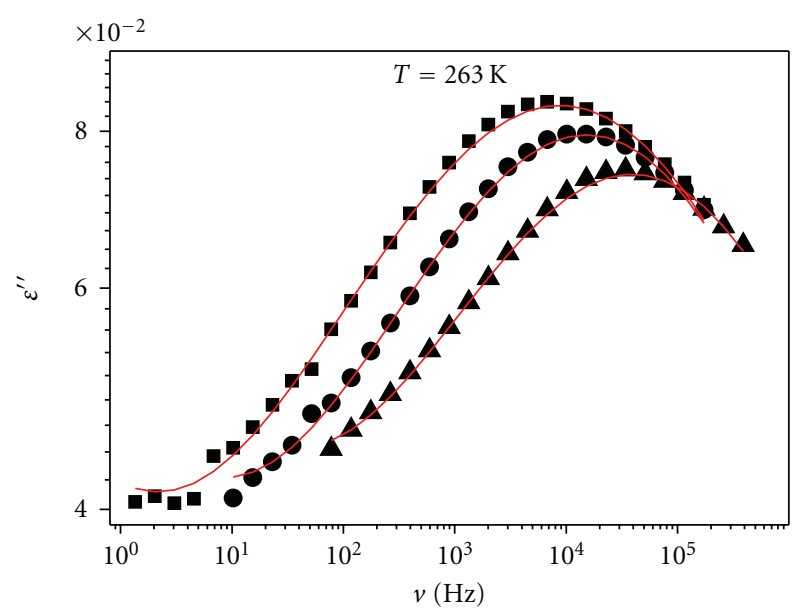

- $P=200 \mathrm{MPa}$

- $P=300 \mathrm{MPa}$

- $P=400 \mathrm{MPa}$

(b)

FIgUre 1: Isothermal loss spectra of (a) PPGE at $T=244 \mathrm{~K}$ and (b) PVAc at $T=263 \mathrm{~K}$, at various pressures in the glassy state. We can see the secondary peak that slightly moves with pressure.

results with JG relaxation of PPGE. We focused on the slower secondary process of PPGE (JG relaxation) and on the one observed in PVAc whose microscopic origin is not clear. We found a non-monotonic behaviour of $\Delta \mathrm{V}_{\beta}$ in secondary relaxation in PVAc.

\section{Experimental}

PPGE, with average molecular weight, MW of $345 \mathrm{~g} / \mathrm{mol}$, and $T_{g}$ around $258 \pm 1 \mathrm{~K}[14]$ and PVAc with MW of $167 \mathrm{Kg} / \mathrm{mol}$ and $T_{g}=310.7 \mathrm{~K}$ were purchased from Sigma-Aldrich. PVAc has interesting structural variations and can be obtained in the atactic and, thus, amorphous form, which is crucial for investigations of the glass transition. PVAc has often been chosen to test the current aspects of various theories related with glass transition phenomena. An ample dipole moment makes PVAc a good candidate for dielectric spectroscopy $[15,16]$. Dielectric measurements were carried out using a dielectric spectrometer (Alpha-Novocontrol) in the frequency interval $10^{-2}-10^{7} \mathrm{~Hz}$. For measurements at ambient pressure, the sample was placed in a parallel plate cell (diameter $=30 \mathrm{~mm}$, gap $=0.1 \mathrm{~mm}$ ) and the temperature control was performed with a precision better than $0.1 \mathrm{~K}$ by using a dry-nitrogen-stream-based system. For measurements at high pressure, the sample was placed in a parallel plate cell (diameter $=20 \mathrm{~mm}$, gap $=0.05 \mathrm{~mm}$ ) which was properly insulated from the external environment and located inside a pressure chamber. Pressure variations (0.1$600 \mathrm{MPa}$ ) were generated by a manual pump and transmitted to the sample through silicon oil. A liquid circulator connected to a jacket and wrapped around the pressure room allowed the control of the temperature (353-233 K) within $\pm 0.1 \mathrm{~K}$.

\section{Results}

Representative isothermal dielectric loss spectra of the investigated glass formers in the glassy state are presented in Figure 1. We obtained dielectric spectra by varying the pressure from $0.1 \mathrm{MPa}$ and up to the maximum value of $600 \mathrm{MPa}$. In both systems we can observe a peak corresponding to the secondary relaxation. In the case of PPGE, we observe at high frequencies a rise in the signal partly due to the presence of a faster relaxation, which, however, is never completely visible with our apparatus for the high-pressure measurements. The $\beta$-relaxation moves to lower frequencies. It is reported that the $\beta$-secondary process of PPGE is of the JG relaxation type [17]. We analyzed spectra in terms of a Cole-Cole equation (CC equation) for the secondary relaxation. As known, the CC erquation is a special case of the Havriliak-Negami equation (HN equation) (1) when the symmetry parameter $(\beta)$ is equal to 1

$$
\varepsilon(\omega)=\varepsilon_{\infty}+\frac{\Delta \varepsilon}{\left[1+\left(i \omega \tau_{\beta}\right)^{1-\alpha}\right]^{\beta}},
$$

where $\varepsilon^{*}(\omega)$ is the complex dielectric constant, $\Delta \varepsilon \equiv \varepsilon_{s}-\varepsilon_{\infty}$ : $\varepsilon_{s}$ and $\varepsilon_{\infty}$ are the "static" and "infinite frequency" dielectric constants, $\omega$ is the angular frequency, $\tau$ is a relaxation constant, and the parameter $\alpha$ is an experimentally determined correction factor. In the case of PPGE, we added a HN equation (1) for fitting the faster secondary relaxation $(\gamma$ relaxation). The shape parameters used in the HN equation for the $\gamma$-relaxation in this system were obtained from spectral fitting at very low temperature and ambient pressure where the $\gamma$-relaxation can be clearly observed. Moreover, a similar procedure was used for fitting to the tail of the structural peak when present in the spectra.

The pressure dependence of secondary relaxation for the different materials with different origins is represented in 


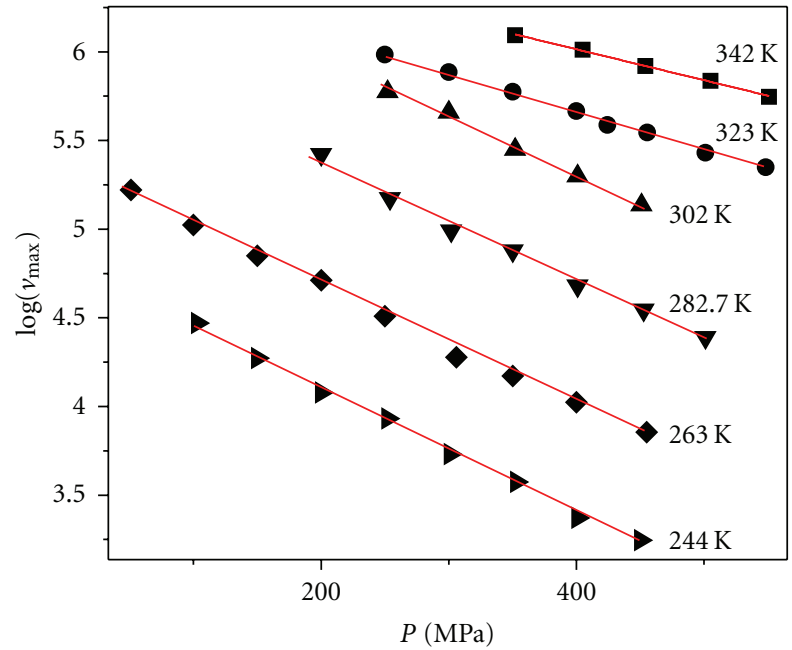

(a)

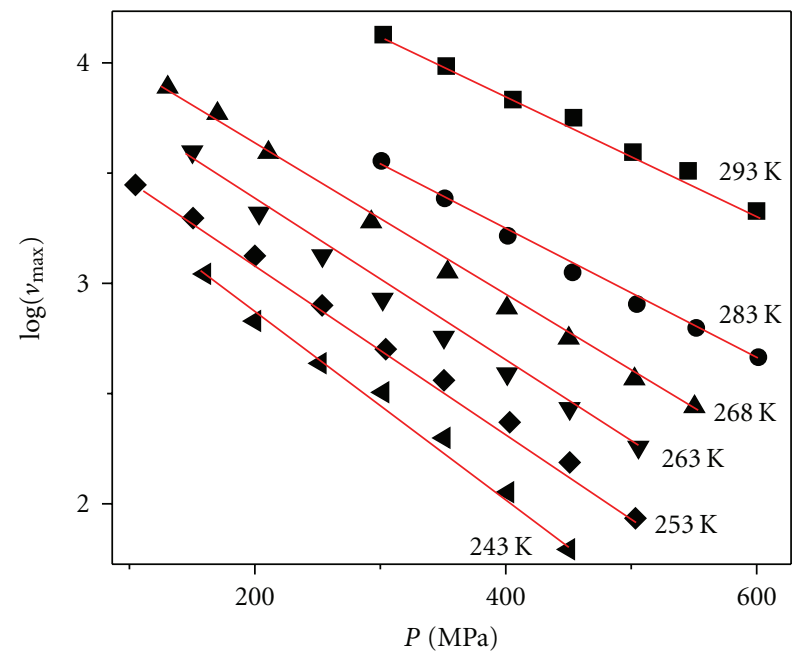

(b)

FIGURE 2: Logarithm of the maximum frequency of the dielectric loss peak of secondary relaxation as a function of pressure at different temperatures (as indicated in the figure) for (a) PPGE, (b) PVAc.

Figure 2. The maximum frequency was calculated by $v_{m}=$ $\left(1 / 2 \pi \tau_{c c}\right)$, where $\tau_{c c}$ is the parameter from fitting with $C C$ function, that is, relaxation time of the $\tau_{\beta},(\beta=1)$ at (1).

The logarithm of the maximum frequency decreases linearly with increasing pressure for both systems. This dependence can be described by the pressure counterpart of the temperature Arrhenius law, $\left[v_{\max }^{\beta}=v_{P=0} \exp \left[-P \Delta \mathrm{V}_{\beta}\right]\right.$ $\left.\left(K_{b} T\right)\right]$. From the analysis of the pressure dependence of the relaxation frequency, we obtained the values of $\Delta V_{\beta}$ (reported in Table 1).

\section{Discussion}

The experimental procedure followed for studying the temperature dependence of $\Delta \mathrm{V}_{\beta}$ in PVAc is represented in Figure 3. A similar procedure was used for PPGE as well. In

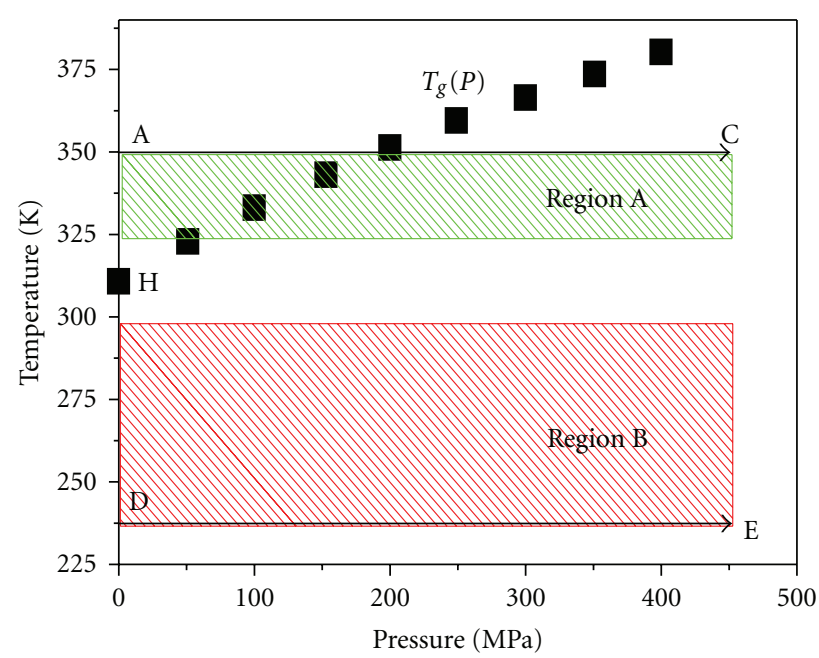

FIgURE 3: Schematic representations of the thermodynamic paths chosen for the investigation of the temperature dependence of $\Delta \mathrm{V}_{\beta}$ in PVAc. Square points show $T_{g}(P)$ line [14], and $\mathrm{AC}$ and DE are isothermal paths.

TABle 1: Parameters describing the pressure dependence of secondary processes.

\begin{tabular}{ccccc}
\hline & $T(\mathrm{~K})$ & $\begin{array}{c}\operatorname{d} \log \left(\nu_{\max }\right) / \mathrm{dP} \\
(1 / \mathrm{MPa})\end{array}$ & $\Delta \mathrm{V}_{\beta}(\mathrm{mL} / \mathrm{mol})$ & $\log \left(\nu_{\max }\right)_{P=0}$ \\
\hline \multirow{4}{*}{ PPGE } & 293 & $(26.1 \pm 1) 10^{-4}$ & $14.7 \pm 0.5$ & $4.92 \pm 0.09$ \\
& 283 & $(29.6 \pm 1) 10^{-4}$ & $16.1 \pm 0.5$ & $4.61 \pm 0.07$ \\
& 263 & $(352 \pm 2) 10^{-5}$ & $18.2 \pm 0.1$ & $4.31 \pm 0.02$ \\
& 253 & $(369 \pm 2) 10^{-5}$ & $18.5 \pm 0.1$ & $4.07 \pm 0.03$ \\
& 243 & $(369 \pm 2) 10^{-5}$ & $18.0 \pm 0.1$ & $3.84 \pm 0.02$ \\
& 342 & $(174 \pm 3) 10^{-5}$ & $17.4 \pm 0.1$ & $3.5 \pm 0.05$ \\
\hline \multirow{4}{*}{ PVAC } & 302 & $(32.8 \pm 1) 10^{-4}$ & $20.6 \pm 0.6$ & $6.6 \pm 0.04$ \\
& 282 & $(31.4 \pm 1) 10^{-4}$ & $19.6 \pm 0.6$ & $6.4 \pm 0.04$ \\
& 263 & $(339 \pm 6) 10^{-5}$ & $18.5 \pm 0.3$ & $5.3 \pm 0.02$ \\
& 244 & $(353 \pm 6) 10^{-5}$ & $17.8 \pm 0.3$ & $4.8 \pm 0.02$ \\
\hline
\end{tabular}

all the cases, the $\beta$-relaxation was investigated in the glassy state; however, in region A the glass was formed by increasing $P$ from the temperature at which the system at ambient pressure is in the liquid state (e.g., line A-C in Figure 3). Instead, in region $\mathrm{B}$, the system is already in the glassy state at ambient pressure (e.g., line D-E in Figure 3).

Figure 4 presents the values of $\Delta \mathrm{V}_{\beta}$ as a function of temperature for PPGE and PVAc (values in Table 1). The activation volume in region $\mathrm{A}$ increases with decreasing temperature for both systems. Most studies about activation volume of $\alpha$-relaxation, $\Delta \mathrm{V}_{\alpha}$, in the liquid and supercooled liquid state evidence that also $\Delta \mathrm{V}_{\alpha}$ increases with decreasing temperature [18]. So, the activation volumes of the secondary relaxation in the region $\mathrm{A}$ have a behaviour similar to that of the $\alpha$-relaxation. Instead, in region $\mathrm{B}$, the activation volume of secondary relaxation is constant or slightly 


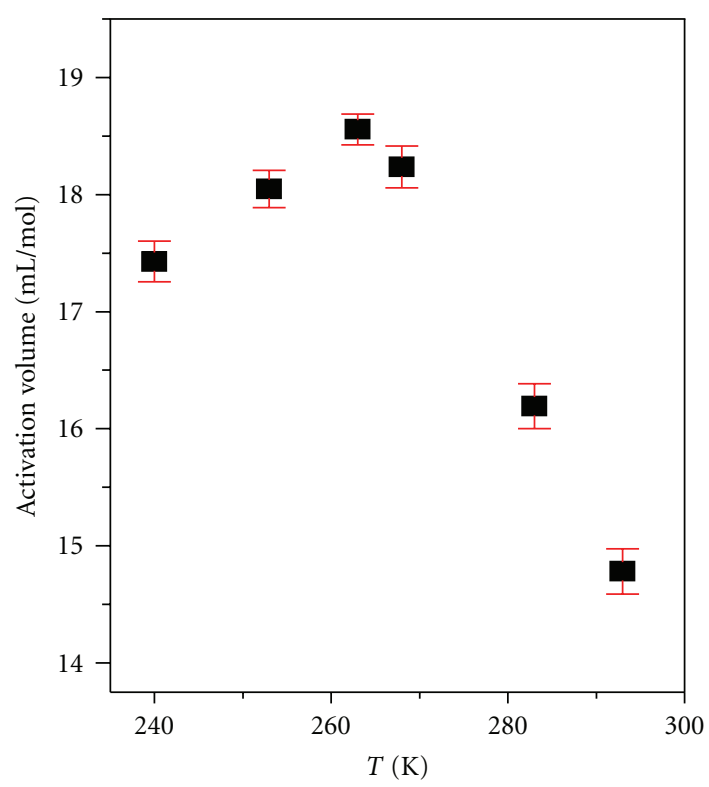

(a)

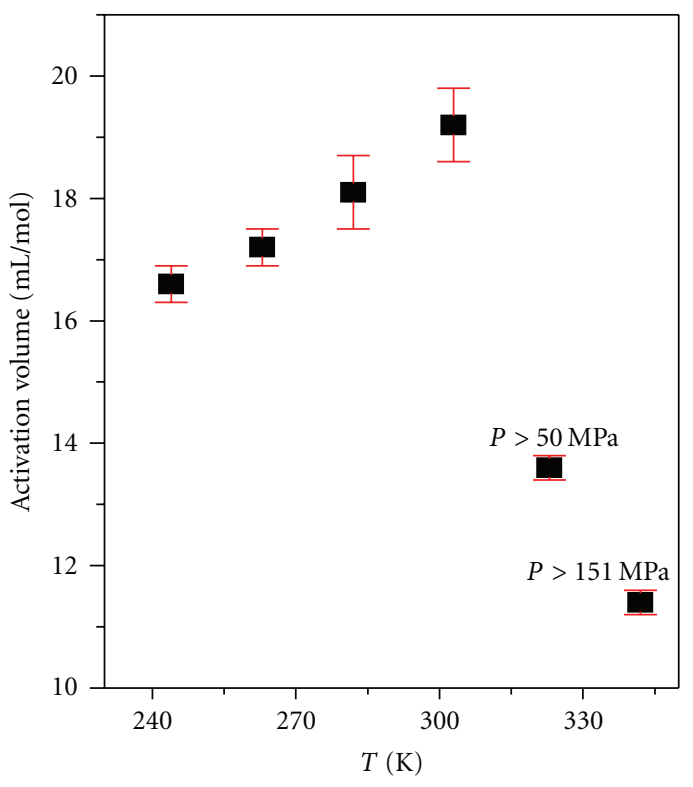

(b)

FIGURE 4: Activation volume of secondary relaxation $\left(\Delta \mathrm{V}_{\beta}\right)$ in the glassy state as a function of temperature for (a) PPGE and (b) PVAc.

decreases with temperature. This result is contrasting with the intuition that decreasing the temperature leads to the reduction of the free volume and consequently to the energy barrier to be overcome and that activation volume should increase. Finally, glass under pressure and temperature has two different regions.

The study on the relation between alpha and secondary relaxation near glass transition has shown that at $T_{g}$ JG relaxation has the same position after different thermodynamic paths (different pressures and temperatures at $T_{g}$ ) and effect of thermodynamic paths comes after the glass transition temperature and pressure inside the glass $[19,20]$. We know, free volume inside the glass is showing a peak at $T_{g}$ and this behaviour is similar to the maximum of activation volume at $T_{g}$ [13]. So, our results show that the non-monotonic behaviour of secondary relaxation inside the glass does not depend on to the type of secondary relaxation, but it depends on the free volume inside the glass.

\section{Conclusions}

We investigated the temperature dependence of the activation volume in PPGE and PVAc, exploring the region where the glass was formed either by compression (region A) or by cooling (region B). In both cases, the temperature dependence is non-monotonic since in region $\mathrm{A}$ the activation volume increases with a decrease of the temperature, but in region $\mathrm{B}$, it decreases with a decrease of the temperature; thus, it seems that we can prepare two different regions. This behaviour does not depend on the type of relaxation.

\section{Acknowledgments}

This work was supported by the University of Sistan and Baluchestan.

\section{References}

[1] C. A. Angell, "Formation of glasses from liquids and biopolymers," Science, vol. 267, no. 5206, pp. 1924-1935, 1995.

[2] L. C. E. Struik, Physical Aging in Amorphous Polymers and Other Materials, Elsevier, Amsterdam, The Netherlands, 1978.

[3] H. W. Bree and J. Heijboer, "The effect of densification on the mechanical properties of amorphous glassy polymers," Journal of Polymer Science, vol. 12, no. 9, pp. 1857-1864, 1974.

[4] A. J. Kovacs, R. A. Stratton, and J. D. Ferry, "Dynamic mechanical properties of polyvinyl acetate in shear in the glass transition temperature range," Journal of Physical Chemistry, vol. 67, no. 1, pp. 152-161, 1963.

[5] J. E. McKinney and M. Goldstein, "PVT relationships for liquid and glassy poly(vinyl acetate)," J Res Natl Bur Stand Sect A Phys Chem, vol. 78, no. 3, pp. 331-353, 1974.

[6] N. B. Olsen, T. Christensen, and J. C. Dyre, " $\beta$ relaxation of nonpolymeric liquids close to the glass transition," Physical Review, vol. 62, no. 3 B, pp. 4435-4438, 2000.

[7] A. Reiser, G. Kasper, and S. Hunklinger, "Effect of pressure on the secondary relaxation in a simple glass former," Physical Review Letters, vol. 92, no. 12, p. 125701, 2004.

[8] M. Paluch, S. Pawlus, S. Hensel-Bielowka et al., "Effect of glass structure on the dynamics of the secondary relaxation in diisobutyl and diisoctyl phthalates," Physical Review, vol. 72, no. 22, p. 224205, 2005.

[9] R. Casalini, M. Paluch, and C. M. Roland, "Dynamic crossover in supercooled liquids induced by high pressure," Journal of Chemical Physics, vol. 118, no. 13, pp. 5701-5703, 2003.

[10] F. Kremer and Andreas Schönhals, Broadband Dielectric Spectroscopy, Springer, Berlin, Germany, 2003.

[11] D. P. Hedvig, Dielectric Spectroscopy of Polymers, John Wiley \& Sons, New York, NY, USA, 1977.

[12] K. L. Ngai and M. Paluch, "Classification of secondary relaxation in glass-formers based on dynamic properties," Journal of Chemical Physics, vol. 120, no. 2, p. 857, 2004. 
[13] S. Sharifi, D. Prevosto, S. Capaccioli, M. Lucchesi, and P. Rolla, "Temperature and pressure dependence of secondary process in an epoxy system ," Journal of Chemical Physics, vol. 134, no. 4, 6 pages, 2011.

[14] R. Casalini, M. Paluch, and C. M. Roland, "Influence of molecular structure on the dynamics of supercooled van der Waals liquids," Physical Review, vol. 67, no. 3, Article ID 031505, pp. 031505/1-031505/6, 2003.

[15] A. Alegr, L. Goitiand, and I. Teller, " $\alpha$-relaxation in the glasstransition range of amorphous polymers. 2 . Influence of physical aging on the dielectric relaxation," J. Colmenero. Macromolecules, vol. 30, p. 3881, 1997.

[16] L. Goitiandi and A. Alegría, "Physical aging of poly(vinyl acetate). A thermally stimulated depolarization current investigation," Journal of Non-Crystalline Solids, vol. 287, p. 237, 2001.

[17] S. Capaccioli, D. Prevosto, K. Kessairi, M. Lucchesi, and P. Rolla, "Relation between the dispersion of $\alpha$-relaxation and the time scale of $\beta$-relaxation at the glass transition," Journal of Non-Crystalline Solids, vol. 353, no. 41-43, pp. 3984-3988, 2007.

[18] M. Paluch, R. Casalini, S. Hensel-Bielowka, and C. M. Roland, "Effect of pressure on the $\alpha$ relaxation in glycerol and xylitol," Journal of Chemical Physics, vol. 116, no. 22, pp. 9839-9844, 2002.

[19] H. W. Starkweather Jr., "Simple and complex relaxations," Macromolecules, vol. 14, no. 5, pp. 1277-1281, 1981.

[20] A. Alegría, L. Goitiandia, I. Tellería, and J. Colmenero, "Dielectric relaxation and physical aging in polar glassy polymers," Journal of Non-Crystalline Solids, vol. 131-133, part 1, pp. 457$461,1991$. 

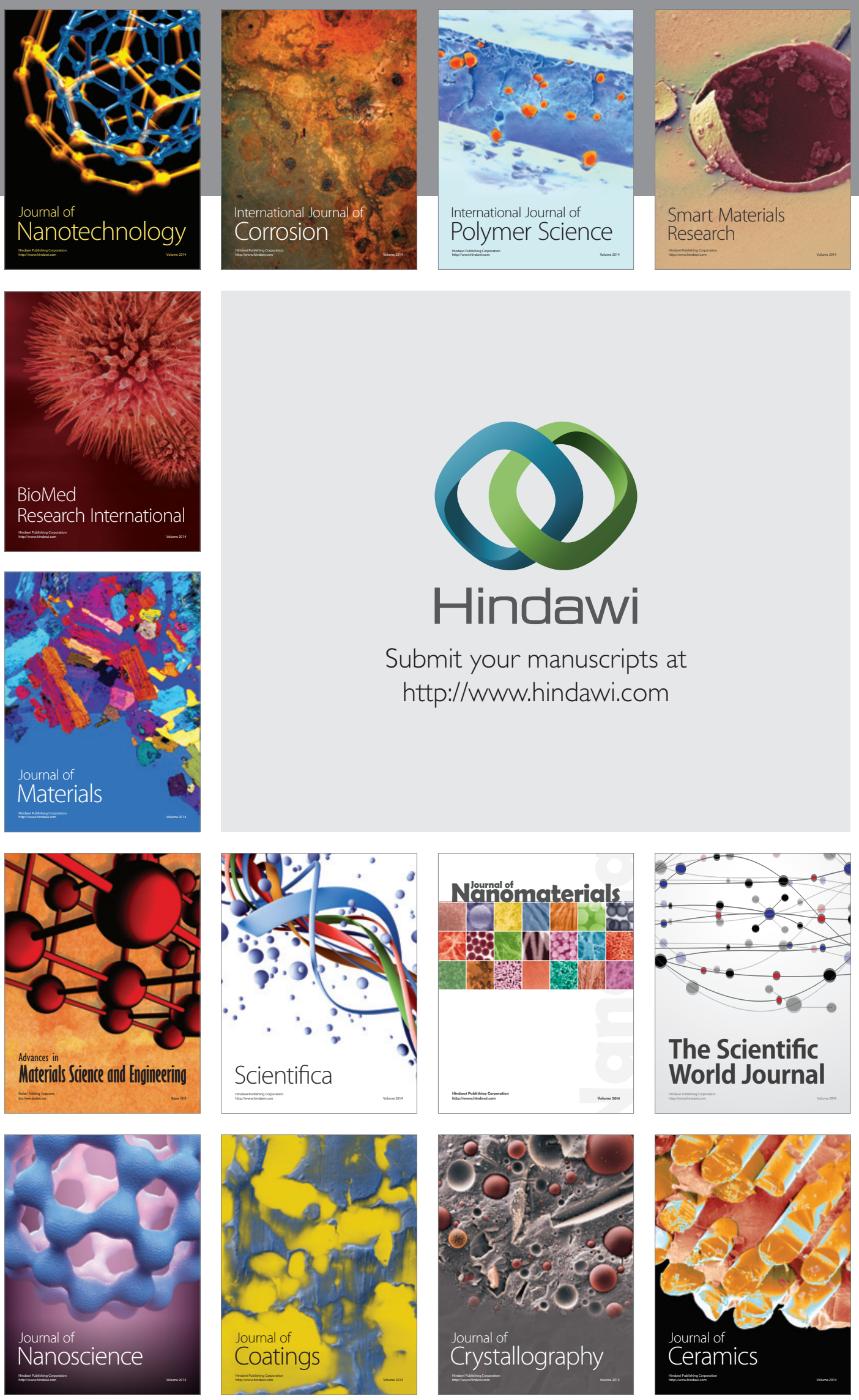

The Scientific World Journal

Submit your manuscripts at

http://www.hindawi.com

\section{World Journal}

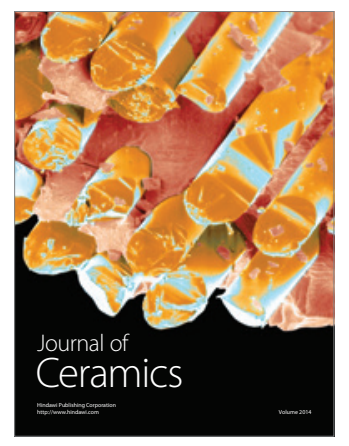

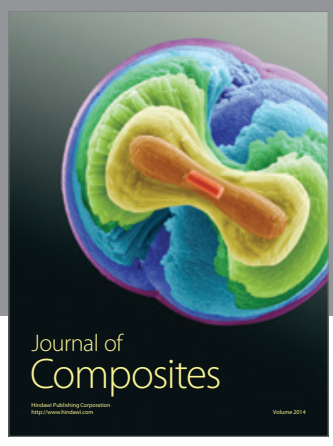
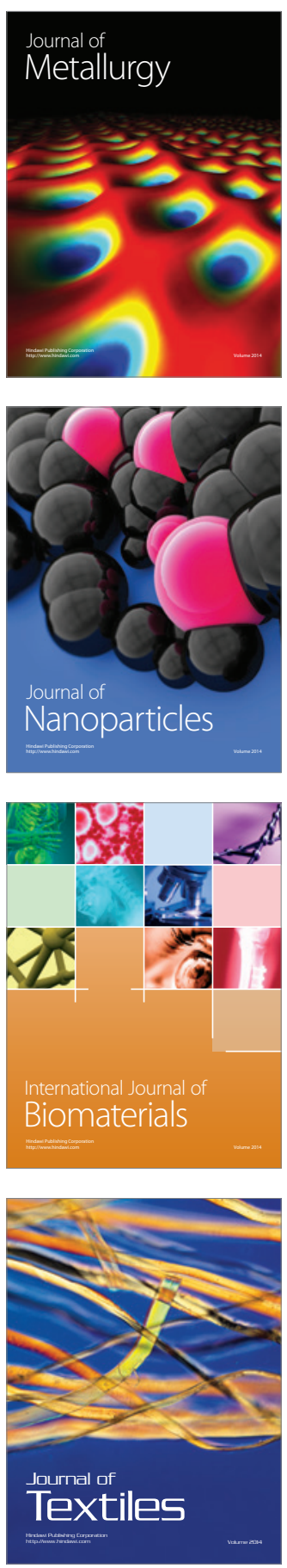